\title{
'Jin Dieyu': A New Cultivar of Lotus with Large, Yellow-green, Duplicate-layered Flowers
}

Ning Shi ${ }^{1}$, Xiaojing Liu ${ }^{1}$, Fengfeng Du, Yajun Chang, and Naiwei Li Institute of Botany, Jiangsu Province and Chinese Academy of Sciences, Nanjing 210014, China

\section{Yuesheng Ding \\ Nanjing Yileen Company, Nanjing 211800, China}

\section{Dongrui $\mathbf{Y a o}^{2}$ \\ Institute of Botany, Jiangsu Province and Chinese Academy of Sciences, Nanjing 210014, China}

Additional index words. Nelumbo nucifera, lotus, duplicate-layered flower, cultivar

Lotus (Nelumbo Adans.) is one of the earliest angiosperms (Du et al., 2016) and comprises only two species, Nelumbo nucifera Gaertn. and Nelumbo lutea (Willd.) Pers, which are herbaceous aquatic perennials that are valued as ornamental plants, especially in landscaping. Nelumbo nucifera is largely distributed throughout Asia and Oceania, with the natural distribution center in China, whereas $N$. lutea is distributed in North America (Wang and Zhang, 2004; Zhang et al., 2011). In China, $N$. nucifera has been cultivated for $\approx 3000$ years resulting in a diversity of floral forms that include single, semidouble, double, duplicate-layered, and thousand-petaled, and flower colors that include white, pink, and red because of the lack of a yellow genotype (Liu et al., 2017). Nelumbo nucifera contrasts with forms of $N$. lutea that are typically characterized by yellow, single flowers with fewer than 20 petals. The creation of interspecific hybrids between the two Nelumbo species has been a longstanding goal for plant breeders and, following several years of breeding from the female parent lotus, 'Friendship Peony', we have bred a duplicate-layered, yellow-flowered hybrid, named 'Jin Dieyu' (Figs. 1 and 2). Here, we describe the breeding process and characters of 'Jin Dieyu', and provide recommendations for its cultivation.

\section{Origin}

The female parent of 'Jin Dieyu' is 'Friendship Peony', which is the progeny of

Received for publication 19 Dec. 2017. Accepted for publication 5 Mar. 2018.

This work was supported by the National Natural Science Foundation of China (Grant No. 31400604), the Natural Science Foundation of Jiangsu Province (Grant No. BK20151370), and Jiangsu Agriculture Science and Technology Innovation Fund (JASTIF), CX (16) 1024

${ }^{1}$ These authors contributed equally to this work. ${ }^{2}$ Corresponding author. E-mail: shuishengzu@ 126.com. interspecific cultivars. We collected seeds from 'Friendship Peony' and bred for selection by open pollination in the Nanjing Yileen lotus garden $\left(32^{\circ} 01^{\prime} \mathrm{N}, 118^{\circ} 36^{\prime} \mathrm{E}\right)$, China. In 2009, we obtained a small to medium size superior individual ('J12') from which we continued to make selections of seedlings. Plants with attractive flowers were further selected as 'Jin Dieyu' in July 2012
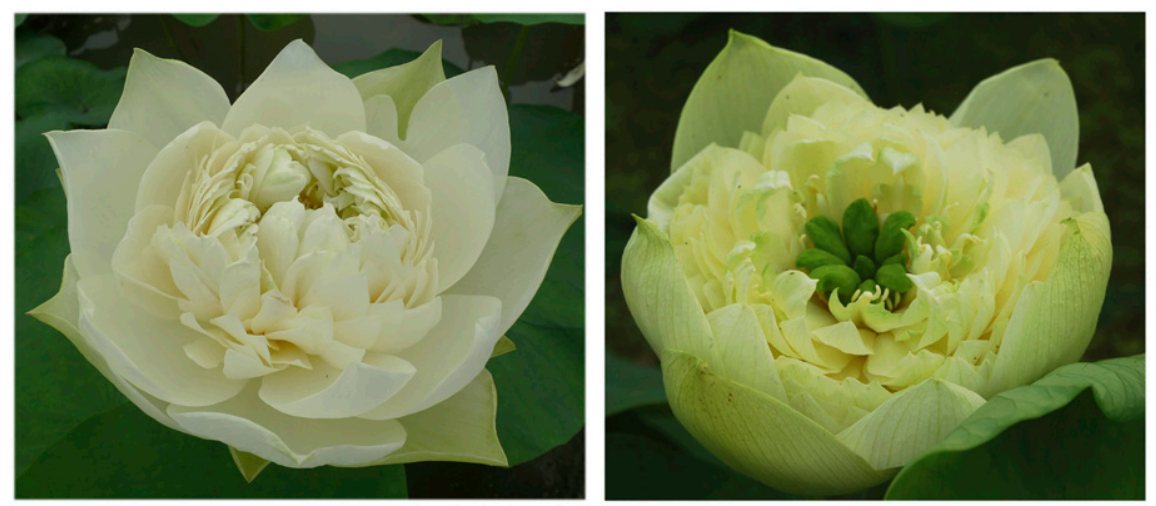

Fig. 1. Flower phenotype of Nelumbo 'Jin Dieyu' and its female parent 'Friendship Peony'. (A) 'Friendship Peony'; (B) 'Jin Dieyu'.
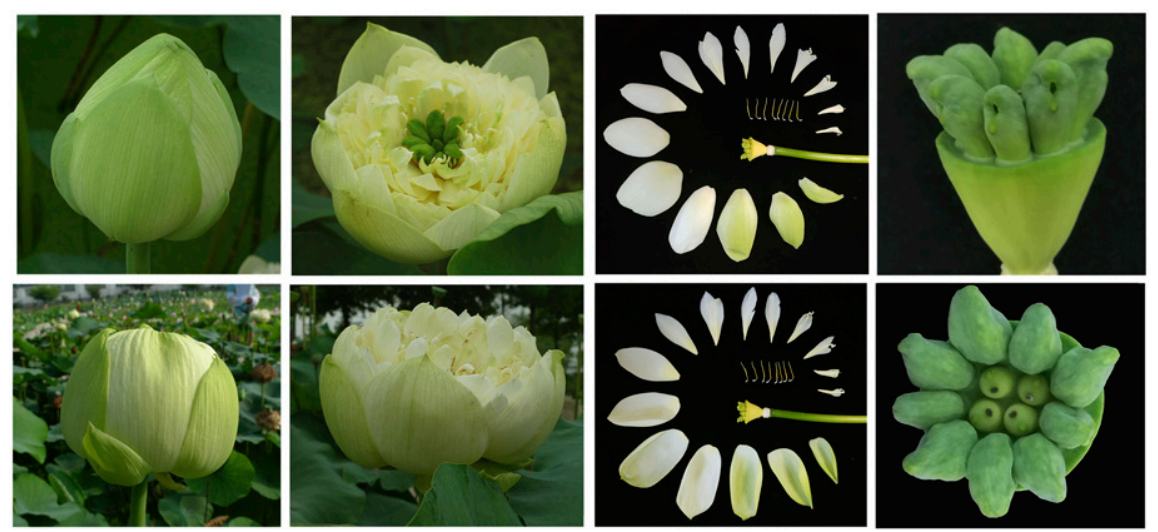

Fig. 2. Horticultural traits of Nelumbo 'Jin Dieyu' plants. (A) flower bud; (B) flower bud before open; (C) flower, top side; (D) flower, lateral side; (E) structure of flowers, adaxial view; (F) structure of flowers, abaxial view; (G) seedpod, lateral side; (H) seedpod, top side. 
Table 1. Flower features of Nelumbo 'Jin Dieyu' and Nelumbo 'Friendship Peony'.

\begin{tabular}{lll}
\hline Nelumbo descriptor & \multicolumn{1}{c}{ Nelumbo 'Jin Dieyu' } & Nelumbo 'Friendship Peony' \\
\hline Flower bud & Green & Green-yellow \\
Flower color & Yellow-green & Yellow \\
Flower form & Double & Double \\
Flower shape & Bowl-shaped & Cup-shaped \\
Petal coloration & Petals of outer layers: base, yellow-green (RHS145C) & Petals of outer layers: green-white (RHS157B) \\
& Middle, yellow-green (RHS 144D) & Petals of inner layers: base, yellow (RHS4C) \\
& Upper, yellow-green (RHS 144B) & Upper, green-yellow (RHS 1D) \\
Dorsal veins on petal & Petals of inner layers: base, green-yellow (RHS 1B) & \\
Color of dorsal vein & Upper, green-yellow (RHS 1D) & Clear \\
Stamens & Clear & Pale yellow-green \\
Carpel & Green & Partially petaloid \\
Top surface of seedpod & Partially petaloid & Normal \\
\hline
\end{tabular}

${ }^{\mathrm{z}}$ Descriptions of color are based on comparison with RHS Color Chart.

Table 2. Plant characteristics of Nelumbo ‘Jin Dieyu' and Nelumbo 'Friendship Peony' grown in open field during summer of 2015 and 2016 in Nanjing, China.

\begin{tabular}{|c|c|c|c|c|c|c|c|}
\hline \multirow[b]{2}{*}{ Cultivar } & \multicolumn{2}{|c|}{ Leaf blade size } & \multirow[b]{2}{*}{ Plant ht } & \multirow[b]{2}{*}{ Flower size (diam) } & \multirow{2}{*}{$\frac{\text { Petal number }}{\text { Total number of petals }}$} & \multicolumn{2}{|c|}{ Outer petal size } \\
\hline & Length & Width & & & & Length $(\mathrm{cm})$ & Width $(\mathrm{cm})$ \\
\hline 'Jin Dieyu' & $45.00 \pm 2.31 \mathrm{a}^{\mathrm{z}}$ & $39.33 \pm 3.53 \mathrm{a}$ & $125.67 \pm 4.06 \mathrm{a}$ & $24.67 \pm 2.60 \mathrm{a}$ & $265.33 \pm 4.10 \mathrm{a}$ & $12.30 \pm 0.89 \mathrm{a}$ & $8.93 \pm 0.64 \mathrm{a}$ \\
\hline 'Jin Dieyu' & $48.17 \pm 1.69 \mathrm{a}$ & $39.83 \pm 1.01 \mathrm{a}$ & $114.00 \pm 2.89 \mathrm{a}$ & $24.43 \pm 2.60 \mathrm{a}$ & $275.00 \pm 10.97 \mathrm{a}$ & $12.63 \pm 0.70 \mathrm{a}$ & $9.17 \pm 0.60 \mathrm{a}$ \\
\hline 'Friendship Peony' & $29.73 \pm 3.47 \mathrm{~b}$ & $26.77 \pm 3.79 \mathrm{~b}$ & $80.00 \pm 9.07 \mathrm{~b}$ & $15.57 \pm 1.62 \mathrm{~b}$ & $209.00 \pm 18.03 \mathrm{~b}$ & $8.67 \pm 0.63 \mathrm{~b}$ & $3.87 \pm 0.38 \mathrm{~b}$ \\
\hline
\end{tabular}

${ }^{\mathrm{z}}$ Different letters indicate significant differences among the treatments according to Duncan's multiple range test $(P<0.05)$.

and Nelumbo 'Friendship Peony' are presented in Table 1.

\section{Performance}

In 2015 and 2016, the performance of newly planted 'Jin Dieyu' and parent 'Friendship Peony' was evaluated in field trials at Nanjing Yileen lotus garden. Rhizomes of 'Jin Dieyu' were separated and planted in the ponds in early April and by mid-April, floating leaves were visible. Standing leaves were growing vigorously in May and then flowering occurred between July and September.

Lotus rhizomes with at least one terminal bud were used as a measure of reproduction and in mid-July. Plant height, number of petals, and sizes of leaf, flower, and outer petals were recorded from five plants for each cultivar. Duncan's multiple range test was used to test for differences in the measures of plant performance between 'Jin Dieyu' and 'Friendship Peony' $(P<$ 0.05 ) in each year.

Overall, we found that 'Jin Dieyu' maintained stability of major ornamental traits and had a similar growth habit to $N$. lutea. 'Jin Dieyu' was larger than its female parent, being 34 and $43 \mathrm{~cm}$ higher than 'Friendship Peony' in 2015 and 2016, respectively, and the mean leaf blade length and width were about 15 and $9 \mathrm{~cm}$ larger, respectively (Table 2). Flowers were also larger in 'Jin Dieyu' than 'Friendship Peony', where they were about $7 \mathrm{~cm}$ in diameter larger, and there were an additional 50 petals in 'Jin Dieyu' in both years (Table 2). The mean length and width of the outer petals of 'Jin Dieyu' was $2.5 \mathrm{~cm}$ and about $4 \mathrm{~cm}$ longer in 2015 and about 4 and $5 \mathrm{~cm}$ in 2016, respectively (Table 2). Seed production in 'Jin Dieyu' was low, because of its petaloid stamens and vesiculated carpels; however, rate of seed set in 'Friendship Peony' was 55\% owing to normal stamen and carpel development.

In October, the leaves started to yellow and the plants entered the withering phase. It was necessary to maintain sufficient water for rhizome growth, and the rhizomes remained in the ponds overwinter, before being separated for cultivation the following spring.

\section{Recommendation}

We suggest that although 'Jin Dieyu' could be successfully cultivated in warm and sunny temperate regions, the production of 'Jin Dieyu' in colder climates would require greenhouse protection.

\section{Availability}

Those interested in 'Jin Dieyu' should contact the corresponding author, Dongrui Yao, at the Institute of Botany, Jiangsu Province and Chinese Academy of Sciences.

\section{Literature Cited}

Du, F.F., X.J. Liu, Y.J. Chang, N.W. Li, P.R. Li, and D.R. Yao. 2016. Analysis on genetic diversity and population structure of lotus cultivars (Nelumbo spp.) based on SSR markers. J. Plant Resources Environ. 25: $9-16$.

Liu, X.J., N.W. Li, F.F. Du, X. Li, Y.J. Chang, N. Shi, Y.S. Ding, and D.R. Yao. 2017. 'Zijin Chuoying': A multicolored and duplicatelayered flowered lotus cultivar. HortScience 52:313-315.

Royal Horticultural Society. 2015. RHS colour chart. Royal Hort. Soc., Westminster, London.

Wang, Q.C. and X.Y. Zhang. 2004. Lotus flower cultivars in China. China For. Publishing House, Beijing, China.

Zhang, X.Y., L.Q. Chen, and Q.C. Wang. 2011. New lotus flower cultivars in China. China For Publishing House, Beijing, China. 